\title{
A Starter Guide to Immunofluorescence Testing in Dermatology
}

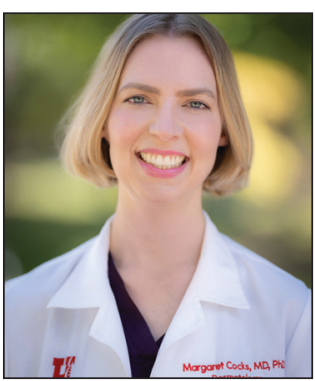

\author{
Margaret Maria Cocks, MD, PhD
}

\section{RESIDENT PEARL}

- Direct immunofluorescence, indirect immunofluorescence, and enzyme-linked immunosorbent assay are important tests for residents to have in their diagnostic tool box, especially when evaluating patients with blistering diseases.
Immunodermatology laboratory testing, including direct immunofluorescence (DIF), indirect immunofluorescence (IIF), and enzymelinked immunosorbent assay (ELISA) are powerful tools that can aide dermatologists when diagnosing autoimmune blistering diseases. Understanding these tests is important to ensure appropriate use and optimum results. This article is intended to serve as a helpful primer for immunofluorescence testing in dermatology, with an overview of the tests available as well as pragmatic tips for optimal biopsy sites and specimen transport.

Cutis. 2021;108:E23-E26.

$\longrightarrow$ irect immunofluorescence (DIF) is the go-to diagnostic test when evaluating vesiculobullous eruptions, connective tissue disease, and vasculitis. This specialized test allows visualization of autoantibodies and their reaction products in the epidermis and dermis (skin) and epithelium and subepithelium (mucosa). Indirect immunofluorescence (IIF) and enzyme-linked immunosorbent assay (ELISA) are additional tests that can help in the diagnosis of autoimmune blistering disease. In the blistering autoimmune diseases, the autoantibodies target components in skin and mucous membranes that are essential for cell-cell and cell-matrix adhesion causing separation within or beneath the epidermis, depending on where the target components are located. This article is intended to serve as a helpful primer for immunofluorescence testing in dermatology, with an overview of the tests available as well as pragmatic tips for optimal biopsy sites and specimen transport.

\section{Direct Immunofluorescence}

Immunofluorescence techniques date back to 1941 when Albert Coons, an American physician, pathologist, and immunologist, fluorescently labelled antibodies to visualize pneumococcal antigens in infected tissues. ${ }^{1-3}$ In dermatology, similar methodology was used to visualize the deposition of immunoglobulins and complement in the skin of patients with systemic lupus erythematosus in 1963. ${ }^{4}$ Basement membrane zone antibodies were first visualized via DIF in bullous pemphigoid in $1967 .^{5}$ This elegant test utilizes specific antibodies labeled with fluorophores that are then incubated with the patient's tissue, ultimately forming antibody-antigen conjugates that can be visualized with a fluorescent microscope. Antibodies usually include IgG, IgM, IgA, fibrinogen, and C3. Some institutions also evaluate for IgG4.

Transport medium is critical for proper evaluation of tissues using DIF. Inappropriate storage of tissue can degrade the antigen and confuse the interpretation of specimens. An acceptable medium for DIF includes Michel transport medium, which allows tissue to be stored for days while being transported at ambient temperature without loss of signal. ${ }^{6,7}$ Zeus medium also can be used and is more readily available. Alternatively, biopsy tissue can be snap frozen using liquid nitrogen. 
Specimens also may be stored on saline gauze but should be analyzed within 24 to 48 hours. ${ }^{8}$ Most importantly, do not place the specimen in formalin; even a brief soak in formalin can greatly alter results, especially when trying to diagnose pemphigus. ${ }^{9}$ Proper transport conditions are critical to prevent autolysis, mitigate putrefaction, and preserve morphology while maintaining antigenicity. ${ }^{10}$

\section{Indirect Immunofluorescence}

Indirect immunofluorescence can be helpful for detecting antibodies circulating in patient serum. Indirect immunofluorescence can be used to help diagnose pemphigoid, pemphigus, epidermolysis bullosa acquisita, bullous lupus erythematosus, and dermatitis herpetiformis. Serum testing also can be a helpful alternative when obtaining tissue is difficult, such as in children.

Indirect immunofluorescence is a 2-part technique that takes a bit longer to assay than DIF. ${ }^{11}$ The first step involves incubating prepared tissue substrates with patient serum. Unlabeled antibodies in the patient serum are allowed to bind to antigens in the substrate tissue for about 30 minutes. Doubling dilutions of patient serum can be performed to titer antibody levels. The second step uses fluorescein-labeled antihuman antibodies to recognize the antigen-antibody conjugates. Normal whole tissues (eg, monkey esophagus for pemphigus vulgaris, rat bladder for paraneoplastic pemphigus, salt-split normal human skin substrate for pemphigoid and epidermolysis bullosa) are the usual substrates for testing. ${ }^{11,12}$ Again, this test requires serum and should be collected in a red-top tube or serum-separator tube. Usually, a minimum of $0.5 \mathrm{~mL}$ is required for testing, but check with your preferred immunodermatology send-out laboratory before collecting. ${ }^{13}$

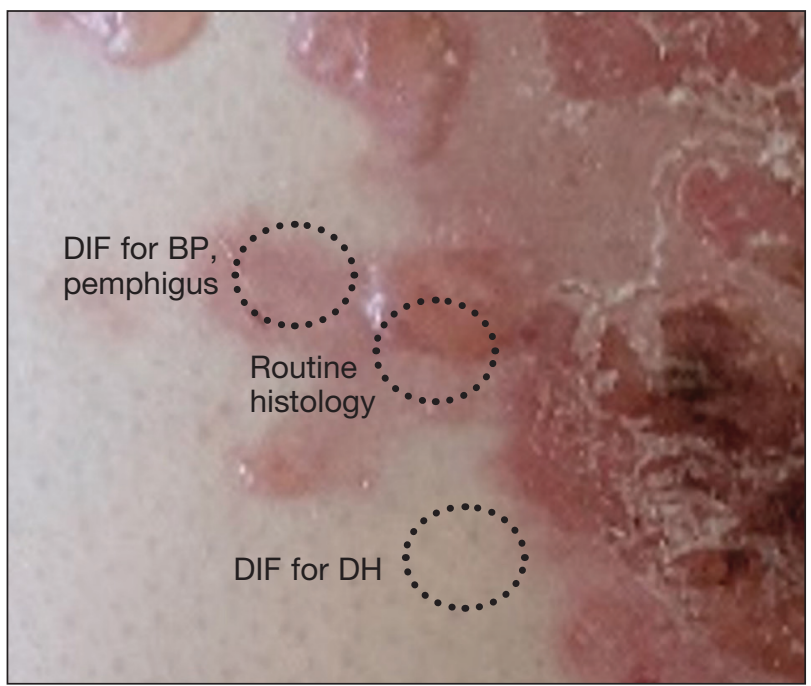

Preferred sites for biopsy specimens for direct immunofluorescence (DIF) in autoimmune bullous disorders. BP indicates bullous pemphigoid; $\mathrm{DH}$, dermatitis herpetiformis.
Indirect immunofluorescence usually involves an initial screening panel using 1 or 2 tissue substrates followed by individual antigen-specific assays that correspond to the clinical suspicion and IIF screening results. ${ }^{11}$ Salt-split skin is used to localize basement membrane zone autoantibodies to either the epidermal (roof) or dermal (floor) side. Although many dermatopathology laboratories offer DIF testing, IIF is more specialized and may be a send-out test at your institution.

\section{Enzyme-linked Immunosorbent Assays}

Another tool in the immunodermatology armamentarium is ELISA. Commercial ELISA systems are available for the detection of autoantibodies against bullous pemphigoid (BP) antigen 180, BP230, type VII collagen, desmoglein (Dsg) 1, Dsg3, and envoplakin. ${ }^{11}$ This test allows semiquantitative measurement of antibody levels and thus can be used to monitor response to treatment or identify relapse and treatment failure. ${ }^{11}$ For example, in BP, significantly increased baseline anti-BP180 IgG levels correlate with 1 -year mortality rates $(P=.001)$ and relapse rates $(P=.041) .{ }^{14,15}$ Numerous additional studies support the observation that monitoring anti-BP180 as a potential marker of disease relapse can be helpful. ${ }^{16,17}$ In pemphigus, the presence or increase of autoantibodies at remission, either anti-Dsg3 or anti-Dsg1, may be a useful tool in predicting disease relapse. ${ }^{18}$ It is important for physicians to be aware of this to be able to offer guidance on prognosis.

\section{Where Should I Biopsy?}

Knowing where to biopsy can be confusing when beginning residency. But the short answer is, it depends. Let your clinical suspicion guide your specimen site. The Figure provides a quick reference for which location will give you the highest yield for a specific diagnosis.

A few cardinal rules should guide which site is biopsied. Avoid obtaining specimens from the lower extremities as much as possible, as this site has been linked with false-negative results, especially in bullous pemphigoid. ${ }^{19,20}$ As a dependent area prone to stasis, this site gets a lot of abuse and inflammatory changes secondary to everyday insults that can theoretically alter DIF findings, especially fibrinogen deposition.

Although tissue sent for hematoxylin and eosin staining should be lesional, biopsy for DIF ideally should not contain a new or active blister, ulcer, erosion, or bulla. Immunoreactants are more likely to be degraded in these areas, and DIF may be falsely negative. ${ }^{21}$

It is worthwhile to briefly discuss the definitions of the terms perilesional and nonlesional. Perilesional skin most frequently refers to skin adjacent to a bulla or vesicle. This skin can be erythematous/inflamed or appear normal. When obtaining tissue for a diagnosis of blistering disease, the general recommendation is to obtain the biopsy from lesional nonbullous skin or perilesional uninvolved skin within $1 \mathrm{~cm}$ of the bulla..22-24 The only exception to 
this is dermatitis herpetiformis, which is best diagnosed on tissue obtained from normal-appearing perilesional skin within $1 \mathrm{~cm}$ of an active lesion. ${ }^{25}$ Additionally, if your patient has oral disease, the recommendation is to obtain the biopsy from nonlesional buccal mucosa, especially if there is desquamative gingivitis. ${ }^{26,27}$

The ideal biopsy size is 4 or $5 \mathrm{~mm}$. If considering both DIF and histopathology, it is best to procure 2 separate specimens. One larger biopsy can be carefully bisected in 2 but often is subject to more handling artifacts, which can affect findings. In the case of 1 biopsy bisected into 2 specimens, the punch should be at least $6 \mathrm{~mm}$. Shave biopsies also can be performed as long as they extend into the reticular dermis. ${ }^{23}$

For vasculitis, biopsies for DIF should be taken from lesions that are less than 24 hours old for highest yield, as the level of tissue immunoreactants tends to decline over time. ${ }^{28}$ This guideline does differ from hematoxylin and eosin specimens sent for evaluation of vasculitis, which ideally should be lesional tissue over 72 hours old. When evaluating for lupus (including subacute cutaneous lupus, discoid lupus, and systemic lupus), DIF is more likely to be positive in well-established, active lesions.

\section{Which Test Should I Order?}

The answer to this question depends, but the use of all 3 tests has a specificity close to $100 \%$ when evaluating for autoantibody-associated diseases. ${ }^{23}$ For autoimmune blistering disease, DIF is considered the diagnostic standard. The sensitivity of DIF for diagnosing BP is in the range of $82 \%$ to $90.5 \%$, while specificity is $98 \%{ }^{29-31}$ Other autoimmune blistering diseases, such as pemphigus or dermatitis herpetiformis, have even higher sensitivities and specificities. Direct immunofluorescence often is used as a screening test, but false negatives do occur. $^{32,33}$ Although rare, false positives also can occur, especially in cases of infection, and should be suspected when there is a lack of clinicopathologic correlation. ${ }^{34}$ If DIF is negative but clinical suspicion remains high, IIF should be ordered to directly evaluate a patient's serum for autoantibodies.

In acute cutaneous lupus, subacute cutaneous lupus, and discoid lupus, DIF of active lesions may be helpful if histopathologic examination of a cutaneous lupus erythematosus lesion is nondiagnostic. However, histopathologic examination of formalin-fixed tissue remains the standard for these diagnoses. In vasculitis, while DIF is not used for diagnosis, it is useful to evaluate for IgA deposition. This is important in adults, as IgA deposition has been associated with a greater risk for developing end-stage renal disease. ${ }^{35}$

\section{Final Thoughts}

This is an overview of the tests available for diagnosing autoimmune blistering diseases. Residents should keep in mind that these tests are just one part of the puzzle when it comes to diagnosing these diseases. Results of DIF, IIF, and ELISA testing should be considered in conjunction with patient history and physical examination as well as histopathologic examination of lesional tissue when evaluating for dermatologic diseases with autoantibodies.

\section{REFERENCES}

1. Arthur G. Albert Coons: harnessing the power of the antibody. Lancet Respir Med. 2016;4:181-182.

2. Coons AH, Creech HJ, Jones RN. Immunological properties of an antibody containing a fluorescent group. Proc Soc Exp Biol Med. 1941;47:200-202.

3. Coons AH, Creech HJ, Jones RN, et al. The demonstration of pneumococcal antigen in tissues by the use of fluorescent antibody. J Immunol. 1942;45:159-170.

4. Burnham TK, Neblett TR, Fine G. The application of the fluorescent antibody technic to the investigation of lupus erythematosus and various dermatoses. J Invest Dermatol. 1963;41:451-456.

5. Jordon RE, Beutner EH, Witebsky E, et al. Basement zone antibodies in bullous pemphigoid. JAMA. 1967;200:751-756.

6. Vaughan Jones SA, Salas J, McGrath JA, et al. A retrospective analysis of tissue-fixed immunoreactants from skin biopsies maintained in Michel's medium. Dermatology. 1994;189(suppl 1):131-132.

7. Kim RH, Brinster NK. Practical direct immunofluorescence. Am J Dermatopathol. 2020;42:75-85.

8. Vodegel RM, de Jong MC, Meijer HJ, et al. Enhanced diagnostic immunofluorescence using biopsies transported in saline. BMC Dermatol. 2004; $4: 10$.

9. Arbesman J, Grover R, Helm TN, et al. Can direct immunofluorescence testing still be accurate if performed on biopsy specimens after brief inadvertent immersion in formalin? J Am Acad Dermatol. 2011;65:106-111.

10. Im K, Mareninov S, Diaz MFP, et al. An introduction to performing immunofluorescence staining. Methods Mol Biol. 2019;1897:299-311.

11. Saschenbrecker S, Karl I, Komorowski L, et al. Serological diagnosis of autoimmune bullous skin diseases. Front Immunol. 2019;10:1974.

12. Baum S, Sakka N, Artsi O, et al. Diagnosis and classification of autoimmune blistering diseases. Autoimmun Rev. 2014;13:482-489.

13. Immunobullous disease panel, epithelial. ARUP Laboratories website. Accessed November 22, 2021. https://tdd.aruplab.com/Tests/ Pub/3001409

14. Monshi B, Gulz L, Piringer B, et al. Anti-BP180 autoantibody levels at diagnosis correlate with 1-year mortality rates in patients with bullous pemphigoid. J Eur Acad Dermatol Venereol. 2020;34:1583-1589.

15. Koga H, Teye K, Ishii N, et al. High index values of enzyme-linked immunosorbent assay for BP180 at baseline predict relapse in patients with bullous pemphigoid. Front Med (Lausanne). 2018;5:139.

16. Fichel F, Barbe C, Joly $\mathrm{P}$, et al. Clinical and immunologic factors associated with bullous pemphigoid relapse during the first year of treatment: a multicenter, prospective study. JAMA Dermatol. 2014;150:25-33.

17. Cai SC, Lim YL, Li W, et al. Anti-BP180 NC16A IgG titres as an indicator of disease activity and outcome in Asian patients with bullous pemphigoid. Ann Acad Med Singap. 2015;44:119-126.

18. Genovese G, Maronese CA, Casazza G, et al. Clinical and serological predictors of relapse in pemphigus: a study of 143 patients [published online July 20, 2021]. Clin Exp Dermatol. doi:10.1111/ced.14854

19. Weigand DA. Effect of anatomic region on immunofluorescence diagnosis of bullous pemphigoid. J Am Acad Dermatol. 1985; 12(2, pt 1):274-278.

20. Weigand DA, Clements MK. Direct immunofluorescence in bullous pemphigoid: effects of extent and location of lesions. J Am Acad Dermatol. 1989;20:437-440.

21. Mutasim DF, Adams BB. Immunofluorescence in dermatology. J Am Acad Dermatol. 2001;45:803-822; quiz 822-824.

22. Sladden C, Kirchhof MG, Crawford RI. Biopsy location for direct immunofluorescence in patients with suspected bullous pemphigoid impacts probability of a positive test result. J Cutan Med Surg. 2014;18:392-396. 
23. Elston DM, Stratman EJ, Miller SJ. Skin biopsy: biopsy issues in specific diseases. J Am Acad Dermatol. 2016;74:1-16; quiz 17-18.

24. Seishima M, Izumi T, Kitajima Y. Antibody to bullous pemphigoid antigen 1 binds to the antigen at perilesional but not uninvolved skin, in localized bullous pemphigoid. Eur J Dermatol. 1999;9:39-42.

25. Zone JJ, Meyer LJ, Petersen MJ. Deposition of granular IgA relative to clinical lesions in dermatitis herpetiformis. Arch Dermatol. 1996;132:912-918.

26. Kamaguchi M, Iwata H, Ujiie I, et al. Direct immunofluorescence using non-lesional buccal mucosa in mucous membrane pemphigoid. Front Med (Lausanne). 2018;5:20.

27. Carey B, Joshi S, Abdelghani A, et al. The optimal oral biopsy site for diagnosis of mucous membrane pemphigoid and pemphigus vulgaris. Br J Dermatol. 2020;182:747-753.

28. Kulthanan K, Pinkaew S, Jiamton S, et al. Cutaneous leukocytoclastic vasculitis: the yield of direct immunofluorescence study. J Med Assoc Thai. 2004;87:531-535.

29. Chaidemenos GC, Maltezos E, Chrysomallis F, et al. Value of routine diagnostic criteria of bullous pemphigoid. Int J Dermatol. 1998;37:206-210.
30. Mysorekar VV, Sumathy TK, Shyam Prasad AL. Role of direct immunofluorescence in dermatological disorders. Indian Dermatol Online J. 2015;6:172-180.

31. Fudge JG, Crawford RI. Bullous pemphigoid: a 10-year study of discordant results on direct immunofluorescence. J Cutan Med Surg. 2018;22:472-475.

32. Sárdy M, Kostaki D, Varga R, et al. Comparative study of direct and indirect immunofluorescence and of bullous pemphigoid 180 and 230 enzyme-linked immunosorbent assays for diagnosis of bullous pemphigoid. J Am Acad Dermatol. 2013;69:748-753.

33. Buch AC, Kumar H, Panicker N, et al. A cross-sectional study of direct immunofluorescence in the diagnosis of immunobullous dermatoses. Indian J Dermatol. 2014;59:364-368.

34. Miller DD, Bhawan J. Bullous tinea pedis with direct immunofluorescence positivity: when is a positive result not autoimmune bullous disease? Am J Dermatopathol. 2013;35:587-594.

35. Cao R, Lau S, Tan V, et al. Adult Henoch-Schönlein purpura: clinical and histopathological predictors of systemic disease and profound renal disease. Indian J Dermatol Venereol Leprol. 2017;83:577-582. 